\title{
NATURAL FREQUENCIES AND CRITICAL VELOCITIES OF FIXED-FIXED LAMINATED CIRCULAR CYLINDRICAL SHELLS CONVEYING FLUIDS
}

\author{
Jeng-Shian Chang and Wen-Jiann Chiou \\ Institute of Applied Mechanics, National Taiwan University, Taipei 10764, Taiwan, Republic of China
}

(Received 20 August 1993)

\begin{abstract}
The natural frequencies and critical velocities of laminated circular cylindrical shells with fixed-fixed ends conveying fluids are studied. Equations of motion are derived by the Hamilton principle under the scope of the Mindlin-type first-order transverse shear deformable cylindrical shell theory. Fluid pressure acting on the wall is obtained through the nonpenetration condition and the assumption of ideal flow. Dynamic characteristic equations are then obtained under the assumption of harmonic motion. Using linear superposition, the natural frequencies corresponding to each flow velocity are found by satisfying dynamic characteristic equation and boundary conditions. Critical velocities are those where the natural frequencies vanish, wherein the static divergence, i.e. buckling, occurs. Numerical examples are presented, in which the parameter studies include stacking angle, length-to-thickness and radius-to-thickness ratios.
\end{abstract}

\section{INTRODUCTION}

Pipes conveying fluids are very commonly used in the petroleum, food, chemical and power industries. The dynamic behavior of such structures has received a great deal of attention over the last 30 years. The decreased natural frequencies with increasing fluid velocity causes susceptible pipes to resonate and eventually failure through fatigue. In the case of larger fluid velocity, the pipes may become unstable (divergence or fiutter). More than a hundred publications have appeared (see the monograph by Chen [1]). Most of the works considered the lateral motion of long pipes, which were treated as beams (for examples, see [2-10]). When the length-to-thickness ratio of a pipe is small, cylindrical shell theory must be applied [11-16].

Recent advances in composite materials has meant that laminated or filament wound cylindrical shells have been used more frequently. However, the relevant works on dynamic behavior of composite cylindrical shells are rather meager. The only exception is Chen and Bert's work [8], which, however, considered only long pipes. Hence, this paper deals with the problem of laminated circular cylindrical shells with fixed-fixed ends conveying fluids, which according to
Holmes [16] can only buckle but not flutter. The natural frequencies at each fluid velocity and the critical velocities corresponding to zero natural frequency, where static divergence occurs, are found for cylindrical shells with various stacking angle, length-to-thickness and radius-to-thickness ratios. Equations of motion are derived by Hamilton's principle under the scope of Mindlin-type first-order transverse shear deformable cylindrical shell theory. Fluid pressure acting on the wall is obtained through the continuity condition and the assumption of the ideal flow. Dynamic characteristic equations are then obtained under the assumption of harmonic motion. Using linear superposition, the natural frequencies corresponding to each flow velocity are found by satisfying the dynamic characteristic equation and boundary conditions. Numerical examples are presented.

\section{GOVERNING EQUATIONS}

\section{Stress-strain relationship}

The stress-strain relationship for the $k$ th layer of the laminated circular cylinder in its material principal axes is [17]

$$
\left\{\begin{array}{c}
\sigma_{1} \\
\sigma_{2} \\
\sigma_{3} \\
\tau_{23} \\
\tau_{13} \\
\tau_{12}
\end{array}\right\}=\left[\begin{array}{cccccc}
Q_{11} & Q_{12} & Q_{13} & 0 & 0 & 0 \\
Q_{12} & Q_{22} & Q_{23} & 0 & 0 & 0 \\
Q_{13} & Q_{23} & Q_{33} & 0 & 0 & 0 \\
0 & 0 & 0 & Q_{44} & 0 & 0 \\
0 & 0 & 0 & 0 & Q_{55} & 0 \\
0 & 0 & 0 & 0 & 0 & Q_{66}
\end{array}\right]\left\{\begin{array}{c}
\epsilon_{1} \\
\epsilon_{2} \\
\epsilon_{3} \\
\gamma_{23} \\
\gamma_{13} \\
\gamma_{12}
\end{array}\right\}
$$


The relations between $Q_{i j}$ and engineering elastic constants are given as

$$
\begin{aligned}
& Q_{11}=E_{1}\left(1-v_{23} v_{32}\right) / S \\
& Q_{22}=E_{2}\left(1-v_{31} v_{13}\right) / S \\
& Q_{33}=E_{3}\left(1-v_{12} v_{21}\right) / S \\
& Q_{44}=G_{23}, \quad Q_{55}=G_{13}, \quad Q_{66}=G_{12} \\
& Q_{12}=\left(v_{21}+v_{31} v_{23}\right) E_{1} / S=\left(v_{12}+v_{32} v_{13}\right) E_{2} / S \\
& Q_{13}=\left(v_{31}+v_{21} v_{32}\right) E_{1} / S=\left(v_{13}+v_{12} v_{23}\right) E_{2} / S \\
& Q_{23}=\left(v_{32}+v_{12} v_{31}\right) E_{2} / S=\left(v_{23}+v_{21} v_{13}\right) E_{3} / S \\
& S=1-v_{12} v_{21}-v_{23} v_{32}-v_{31} v_{13}-2 v_{21} v_{13} v_{32} \\
& v_{i j}=-\epsilon_{j} / \epsilon_{i} .
\end{aligned}
$$

Under the coordinate transformation from the local material principal axes $1-2-3$ into the global $x-y-z$ axes, the transformed stress-strain relation can be shown as

\section{Moderately thick cylindrical shell theory}

Consider a circular shell. The cylindrical shell theory used in this study is based on the improved (Flügge) theory [18]. The following Mindlin-type displacement field is adopted

$$
\begin{aligned}
& u=u^{0}=z u^{a} \\
& v=v^{0}+z v^{a} \\
& w=w^{0} .
\end{aligned}
$$

The related linear strains can be shown to be

$$
\begin{aligned}
\epsilon_{x} & =\epsilon_{x}^{0}+z \chi_{x} \\
\epsilon_{0} & =\left(\epsilon_{0}^{0}+z \chi_{0}\right) /(1+z / a) \\
\gamma_{x 0} & =\left(\beta_{x}^{0}+z \beta_{x}^{a}\right)+\left(\beta_{0}^{0}+z \beta_{0}^{a}\right) /(1+z / a) \\
\gamma_{x z} & =\mu_{x}^{0} \\
\gamma_{0 z} & =\mu_{0}^{0} /(1+z / a) \\
\epsilon_{z} & =0
\end{aligned}
$$

$$
\left\{\begin{array}{c}
\sigma_{x} \\
\sigma_{y} \\
\sigma_{z} \\
\tau_{y z} \\
\tau_{x z} \\
\tau_{x y}
\end{array}\right\}=\left[\begin{array}{cccccc}
\bar{Q}_{11} & \bar{Q}_{12} & \bar{Q}_{13} & 0 & 0 & \bar{Q}_{16} \\
\bar{Q}_{12} & \bar{Q}_{22} & \bar{Q}_{23} & 0 & 0 & \bar{Q}_{26} \\
\bar{Q}_{13} & \bar{Q}_{23} & \bar{Q}_{33} & 0 & 0 & \bar{Q}_{36} \\
0 & 0 & 0 & \bar{Q}_{44} & \bar{Q}_{45} & 0 \\
0 & 0 & 0 & \bar{Q}_{45} & \bar{Q}_{55} & 0 \\
\bar{Q}_{16} & \bar{Q}_{26} & \bar{Q}_{36} & 0 & 0 & \bar{Q}_{66}
\end{array}\right]\left\{\begin{array}{c}
\epsilon_{x} \\
\epsilon_{y} \\
\epsilon_{z} \\
\gamma_{y z} \\
\gamma_{x z} \\
\gamma_{x y}
\end{array}\right\}
$$

In this work, the usual assumption of $\sigma_{z}=0$ is made, then eliminating $\epsilon_{z}$ from eqn (1) yields

$$
\left\{\begin{array}{c}
\sigma_{x} \\
\sigma_{y} \\
\tau_{y z} \\
\tau_{x z} \\
\tau_{x y}
\end{array}\right\}=\left[\begin{array}{ccccc}
\bar{Q}_{11}^{\prime} & \bar{Q}_{12}^{\prime} & 0 & 0 & 0 \\
\bar{Q}_{11}^{\prime} & \bar{Q}_{22}^{\prime} & 0 & 0 & 0 \\
0 & 0 & \bar{Q}_{44} & 0 & 0 \\
0 & 0 & 0 & \bar{Q}_{55} & 0 \\
0 & 0 & 0 & 0 & \bar{Q}_{66}
\end{array}\right\}\left\{\begin{array}{c}
\epsilon_{x} \\
\epsilon_{y} \\
\gamma_{y z} \\
\gamma_{x z} \\
\gamma_{x y}
\end{array}\right\},
$$

where

$$
\begin{aligned}
& \bar{Q}_{x x}^{\prime}=\bar{Q}_{x x}-\frac{\bar{Q}_{13}^{2}}{\bar{Q}_{33}} \\
& \bar{Q}_{x y}^{\prime}=\bar{Q}_{x y}-\bar{Q}_{13} \bar{Q}_{23} / \bar{Q}_{33} \\
& \bar{Q}_{y y}^{\prime}=\bar{Q}_{y y}-\frac{\bar{Q}_{23}^{2}}{\bar{Q}_{33}}
\end{aligned}
$$

Equation (4) is the extended version of conventional plane-stress reduced stress-strain relationship. in which $a$ is the mean radius and

$$
\begin{aligned}
& \epsilon_{x}^{0}=\frac{\partial u^{0}}{\partial x} \\
& \epsilon_{\theta}^{0}=\frac{1}{a}\left(\frac{\partial v^{0}}{\partial \theta}+w^{0}\right) \\
& \chi_{x}=\frac{\partial u^{a}}{\partial x} \\
& \chi_{\theta}^{0}=\frac{1}{a} \frac{\partial v^{a}}{\partial \theta} \\
& \mu_{x}^{0}=\frac{\partial w^{0}}{\partial x}+u^{a} \\
& \mu_{0}^{0}=\frac{1}{a}\left(\frac{\partial w^{0}}{\partial \theta}-v^{0}\right)+v^{a} \\
& \beta_{x}^{0}=\frac{\partial v^{0}}{\partial x} \\
& \beta_{\theta}^{0}=\frac{1}{a} \frac{\partial u^{0}}{\partial \theta}
\end{aligned}
$$




$$
\begin{aligned}
& \beta_{x}^{a}=\frac{\partial v^{a}}{\partial x} \\
& \beta_{0}^{a}=\frac{1}{a} \frac{\partial u^{a}}{\partial \theta}
\end{aligned}
$$

The equation of motion can be obtained through the application of Hamilton's principle. Let $\rho_{s}$ denote the density of the shell. The kinetic energy $T$, strain energy $V$ and external work $W$ done by internal fluid pressure $p^{*}$ can be written as follows:

$$
\begin{gathered}
T=\frac{\rho_{s}}{2} \int_{0}^{L} \int_{-\pi}^{\pi} \int_{-h / 2}^{h / z}\left(\dot{u}^{2}+\dot{v}^{2}+\dot{w}^{2}\right) \\
\times(1+z / a) a \mathrm{~d} x \mathrm{~d} \theta \mathrm{d} z \\
V=\frac{1}{2} \int_{0}^{L} \int_{-\pi}^{\pi} \int_{-h / 2}^{h / 2}\left(\sigma_{i j} \epsilon_{i j}\right) \\
\times(1+z / a) a \mathrm{~d} x \mathrm{~d} \theta \mathrm{d} z, \quad i, j=x, r, \theta \\
W=\int_{0}^{L} \int_{-\pi}^{\pi} p^{*} w a \mathrm{~d} x \mathrm{~d} \theta .
\end{gathered}
$$

Thus, according to the Hamilton principle one can write

$$
\delta \int_{t_{0}}^{h} \int_{0}^{L} \int_{-\pi}^{\pi} \int_{-h / 2}^{h / 2}(T-V+W) \mathrm{d} V \mathrm{~d} t=0
$$

which can be shown to lead to the set of equations of motion

$$
\begin{aligned}
\delta \bar{u}^{0}: \frac{\partial N_{x}}{\partial x}+\frac{\partial N_{\theta x}}{\partial \theta} & =a^{2} h \rho_{s}\left(a \frac{\partial^{2} \bar{u}^{0}}{\partial \bar{t}^{2}}+\alpha \frac{\partial^{2} \bar{u}^{a}}{\partial \bar{t}^{2}}\right) \\
\delta \bar{u}^{a}: \frac{\partial M_{x}}{\partial x}+\frac{\partial M_{0 x}}{\partial \theta}-a Q_{x} & =a^{3} h \alpha \rho_{s}\left(a \frac{\partial^{2} \bar{u}^{0}}{\partial \bar{t}^{2}}+\frac{\partial^{2} \bar{u}^{a}}{\partial \bar{t}^{2}}\right) \\
\delta \bar{v}^{0}: \frac{\partial N_{x \theta}}{\partial x}+\frac{\partial N_{0}}{\partial \theta}+Q_{0} & =a^{2} h \rho_{s}\left(a \frac{\partial^{2} \bar{v}^{0}}{\partial \bar{t}^{2}}+\alpha \frac{\partial^{2} \bar{v}^{a}}{\partial \bar{t}^{2}}\right) \\
\delta \bar{v}^{a}: \frac{\partial M_{x} \theta}{\partial x}+\frac{\partial M_{0}}{\partial \theta}-a Q_{0} & =a^{3} h \alpha \rho_{s}\left(a \frac{\partial^{2} \bar{v}^{0}}{\partial \bar{t}^{2}}+\frac{\partial^{2} \bar{v}^{a}}{\partial \bar{t}^{2}}\right) \\
\delta \bar{w}^{0}: \frac{\partial Q_{x}}{\partial x}+\frac{\partial Q_{0}}{\partial \theta}-N_{0} & =a^{3} h \rho_{s} \frac{\partial^{2} \bar{w}^{0}}{\partial \bar{t}^{2}}+a p^{*}
\end{aligned}
$$

and the set of associated boundary conditions

$$
\begin{aligned}
N_{x} \delta \bar{u}^{0} & =0 \\
N_{x 0} \delta \bar{v}^{0} & =0 \\
M_{x} \delta \bar{u}^{a} & =0 \\
M_{x 0} \delta \bar{v}^{a} & =0 \\
Q_{x} \delta \bar{w}^{0} & =0
\end{aligned}
$$

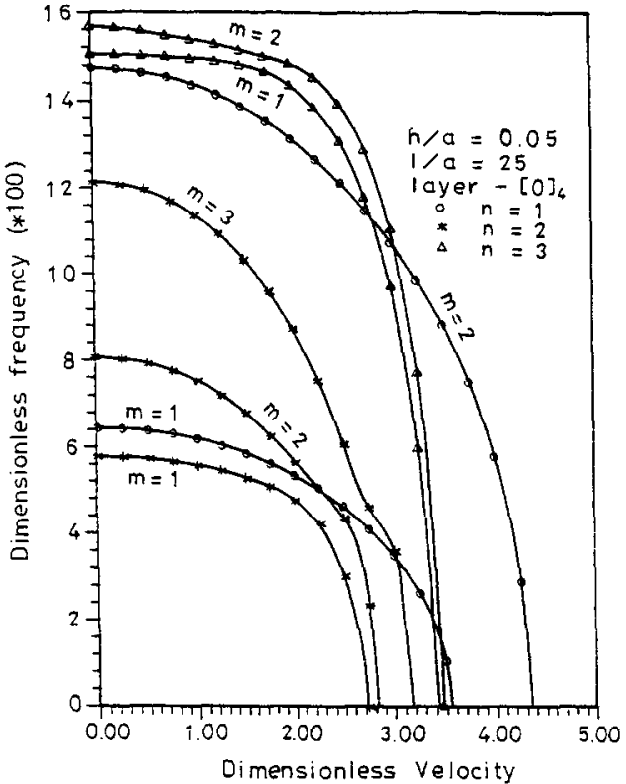

Fig. 1. Relationship of the dimensionless natural frequency vs dimensionless velocity of a $\left[(0)_{4}\right]$ laminated cylindrical shell with thickness-to-radius ratio $h / a=0.05$.

in which $\alpha=h^{2} /\left(12 a^{2}\right)$, and

$$
\begin{aligned}
& N_{x}=\int_{-h / 2}^{h / 2} \sigma_{x}\left(1+\frac{z}{a}\right) \mathrm{d} z \\
& N_{x 0}=\int_{-h / 2}^{h / 2} \tau_{x 0}\left(1+\frac{z}{a}\right) \mathrm{d} z \\
& Q_{x}=\int_{-h / 2}^{h / 2} \tau_{x r}\left(1+\frac{z}{a}\right) \mathrm{d} z
\end{aligned}
$$

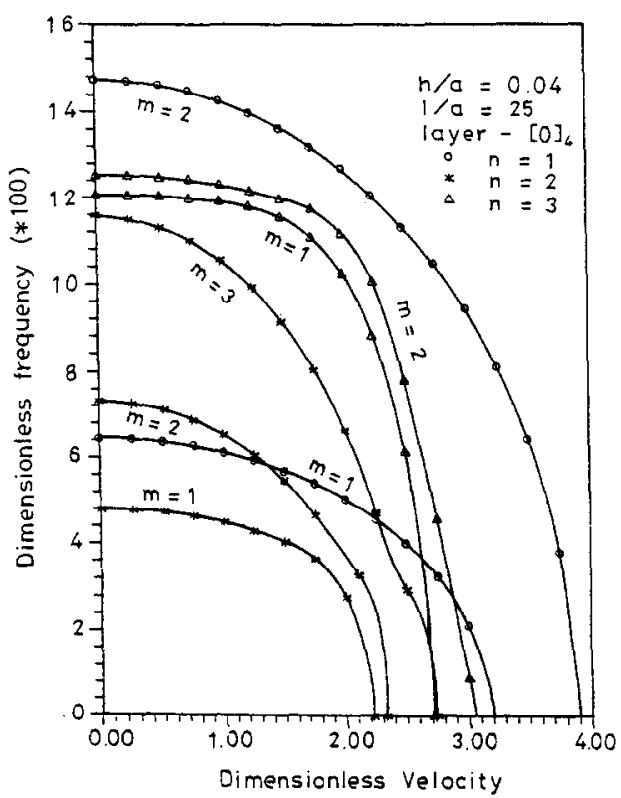

Fig. 2. Relationship of the dimensionless natural frequency vs dimensionless velocity of a $\left[(0)_{4}\right]$ laminated cylindrical shell with thickness-to-radius ratio $h / a=0.04$. 


$$
\begin{aligned}
M_{x} & =\int_{-h / 2}^{h / 2} \sigma_{x} z\left(1+\frac{z}{a}\right) \mathrm{d} z \\
M_{x \theta} & =\int_{-h / 2}^{h / 2} \tau_{x \theta} z\left(1+\frac{z}{a}\right) \mathrm{d} z \\
N_{\theta} & =\int_{-h / 2}^{h / 2} \sigma_{\theta} \mathrm{d} z \\
N_{\theta x} & =\int_{-h / 2}^{h / 2} \tau_{\theta} \mathrm{d} z \\
Q_{x} & =\int_{-h / 2}^{h / 2} \tau_{x r} \mathrm{~d} z \\
M_{\theta} & =\int_{-h / 2}^{h / 2} \sigma_{\theta} \mathrm{d} z \\
M_{\theta x} & =\int_{-h / 2}^{h / 2} \tau_{x \theta} \mathrm{d} z .
\end{aligned}
$$

Only inviscid and incompressible fluid is considered in this work. Thus the velocity field of fluid can be expressed as

$$
\begin{aligned}
& v_{x}=U+\frac{\partial \psi}{\partial x} \\
& v_{0}=\frac{1}{r} \frac{\partial \psi}{\partial \theta} \\
& v_{r}=\frac{\partial \psi}{\partial r}
\end{aligned}
$$

in which $U$ is the uniform (unperturbed) fluid velocity along the axial direction and $\psi$ is the perturbed velocity potential. Let $\rho_{i}$ denote the density of the fluid. The fluid pressure $p^{*}$ can be found through the potential flow theory and the nonpenetration condition at the wall, which turns out to be [14]

$$
\begin{aligned}
p^{*}(x, \theta, t)= & -\left.\rho_{i} \frac{f(a)}{f^{\prime}(a)}\left[\frac{\partial}{\partial t}+u \frac{\partial}{\partial x}\right]^{2} w\right|_{r=a} \\
= & -\frac{\rho_{i}}{n+\lambda I_{n+1}(\lambda) / I_{n}(\lambda)} \\
& \times\left.\left[\frac{\partial}{\partial t}+u \frac{\partial}{\partial x}\right]^{2} w\right|_{r=a}
\end{aligned}
$$

in which $I_{n}$ is the modified Bessel function of the first kind, order $n$.

To find the natural frequencies, we assume the following harmonic forms of the displacement field

$$
\begin{aligned}
& u^{0}=A \exp \left[i\left(\frac{\lambda}{a} x+n \theta+\omega t\right)\right. \\
& u^{a}=B \exp \left[i\left(\frac{\lambda}{a} x+n \theta+\omega t\right)\right. \\
& v^{0}=C \exp \left[i\left(\frac{\lambda}{a} x+n \theta+\omega t\right)\right.
\end{aligned}
$$

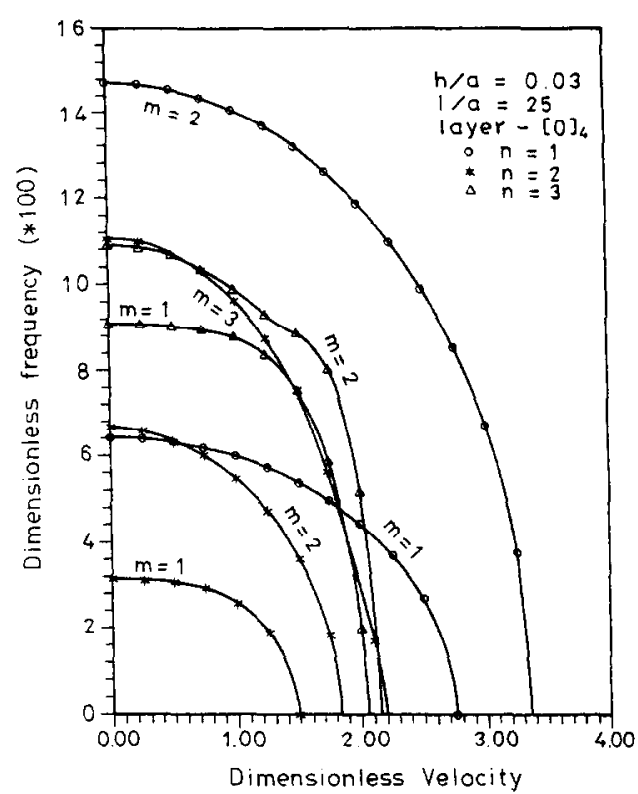

Fig. 3. Relationship of the dimensionless natural frequency vs dimensionless velocity of a $\left[(0)_{4}\right]$ laminated cylindrical shell with thickness-to-radius ratio $h / a=0.03$.

$$
\begin{aligned}
& v^{a}=D \exp \left[i\left(\frac{\lambda}{a} x+n \theta+\omega t\right)\right. \\
& w^{0}=E \exp \left[i\left(\frac{\lambda}{a} x+n \theta+\psi t\right)\right.
\end{aligned}
$$

in which $\lambda, n$ and $\omega$ are respectively the longitudinal wavenumber, circumferential wavenumber and

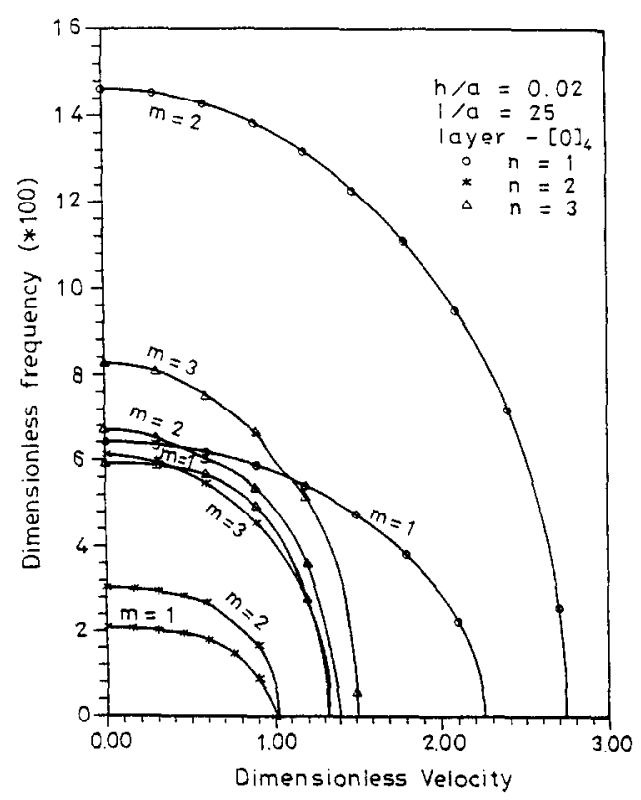

Fig. 4. Relationship of the dimensionless natural frequency vs dimensionless velocity of a $\left[(0)_{4}\right]$ laminated cylindrical shell with thickness-to-radius ratio $h / a=0.02$. 
natural frequency. Now define the following dimensionless quantities

$$
\begin{aligned}
& \bar{x}=x / a \\
& \bar{u}_{0}=u_{0} / a \\
& \bar{u}_{a}=u_{a} \\
& \bar{v}_{0}=v_{0} / a \\
& \bar{v}_{a}=v_{a} \\
& \bar{w}_{0}=w_{0} / a \\
& \bar{t}=t / T_{0} \\
& \Omega=\omega / \omega_{0} \\
& \bar{U}=U / U_{0} \\
& T_{0}=a \sqrt{\rho_{s} / E_{0}} \\
& \omega_{0}=\frac{1}{a} \sqrt{E_{0} / \rho_{s}} \\
& U_{0}=\sqrt{E_{0} / \rho_{s}} .
\end{aligned}
$$

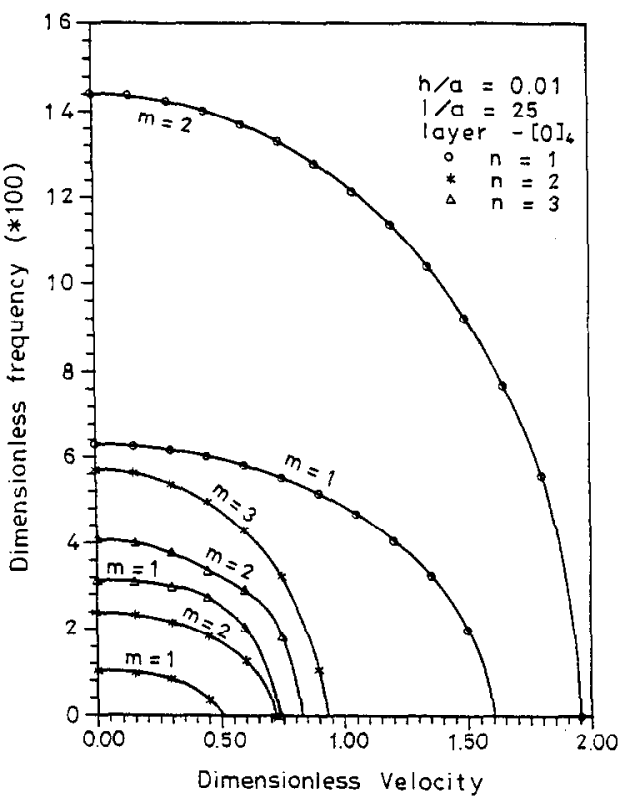

Fig. 5. Relationship of the dimensionless natural frequency vs dimensionless velocity of a $\left[(0)_{4}\right]$ laminated cylindrical shell with thickness-to-radius ratio $h / a=0.01$.

the above homogeneous set of equations must vanish, which can formally be written as

$$
f\left(\lambda, \Omega, \bar{U}, n ; \bar{Q}_{i j}, l / h, a / h\right)=0 .
$$

Given a dimensionless fluid velocity $\bar{U}$ and circumferential wavenumber $n$, one then solves the above equation to yield a set of $\lambda_{j}$ for any estimated frequency $\Omega$. The correct $\Omega$ is the one that the deformation of the cylindrical shell formed by the corresponding set of $\lambda_{j}$ must satisfy the appropriate set of boundary conditions (14). To this end, the true deformation of the cylindrical shells can be written, by mode expansion, as

$$
\begin{aligned}
& u^{0}=\sum_{j=1}^{\infty} A_{j} \exp \left[i\left(\frac{\lambda_{j}}{a} \bar{x}+n \theta+\Omega \bar{t}\right)\right. \\
& u^{a}=\sum_{j=1}^{\infty} B_{j} \exp \left[i\left(\frac{\lambda_{j}}{a} \bar{x}+n \theta+\Omega \bar{t}\right)\right.
\end{aligned}
$$

Substituting the equations (7), (17)-(19) and the stress-strain relations of each orthotropic layer [15] into (13), one can obtain the following dynamic characteristic equations

$$
\int_{-h / 2}^{h / 2}\left[\begin{array}{lllll}
a_{11} & a_{12} & a_{13} & a_{14} & a_{15} \\
a_{21} & a_{22} & a_{23} & a_{24} & a_{25} \\
a_{31} & a_{32} & a_{33} & a_{34} & a_{35} \\
a_{41} & a_{42} & a_{43} & a_{44} & a_{45} \\
a_{51} & a_{52} & a_{53} & a_{54} & a_{55}
\end{array}\right] \mathrm{d} z\left\{\begin{array}{l}
A \\
B \\
C \\
D \\
E
\end{array}\right\}=\{0\}
$$

Details of $a_{i j}$ are given in the Appendix. To obtain nontrivial solutions, the determinant, denoted as $f$, of

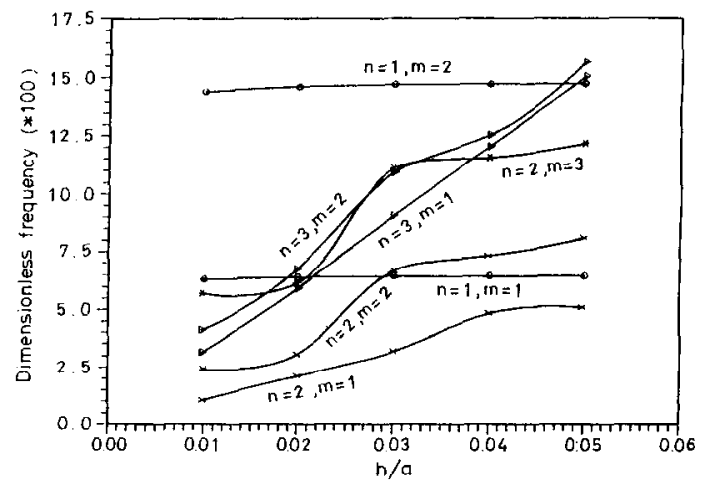

Fig. 6. Effect of $h / a$ on the dimensionless frequencies of a $\left[(0)_{4}\right]$ laminated cylindrical shell with zero fluid velocity. 


$$
\begin{aligned}
& v^{0}=\sum_{j=1}^{\infty} C_{j} \exp \left[i\left(\frac{\lambda_{j}}{a} \bar{x}+n \theta+\Omega \bar{t}\right)\right. \\
& v^{a}=\sum_{j=1}^{\infty} D_{j} \exp \left[i\left(\frac{\lambda_{j}}{a} \bar{x}+n \theta+\Omega \bar{t}\right)\right. \\
& w^{0}=\sum_{j=1}^{\infty} E_{j} \exp \left[i\left(\frac{\lambda_{j}}{a} \bar{x}+n \theta+\Omega \bar{t}\right) .\right.
\end{aligned}
$$

The boundary conditions specified by eqn (14) should be able to be satisfied by the above equations if the correct set of $\lambda_{j}$, which depends on the dimensionless frequency $\Omega$, is used. If so, then the estimated value of $\Omega$ is correct. Otherwise, iteration is necessary to find the correct $\Omega$. It should be noted that the relations between modal components $A_{j}$, $B_{j}, C_{j}, D_{j}$ can be related to $E_{j}$, corresponding to the eigenvalue $\lambda_{j}$, by solving eqn (20). In other words, for each longitudinal mode, there is only one unknown in the eigenvector, say $E_{j}$. Now since there are only 10 boundary conditions, 10 lowest values of $\lambda_{j}$ can be picked from the solution of eqn (21). Thus the substitution of (22) into (14) will result in

$$
[B]_{10 \times 10}\{E\}_{10}=\{0\} .
$$

The satisfaction can be reached by setting the determinant of $[B]$ to zero.

\section{NUMERICAL RESULTS AND DISCUSSION}

The following dimensionless material constants for a single layer, in its material principal axes, are used in the numerical calculation, in this paper

$$
\begin{aligned}
E_{1} / E_{0} & =21, \quad E_{2} / E_{0}=E_{3} / E_{0}=1.7 \\
G_{12} / E_{0} & =G_{13} / E_{0}=0.65, \quad G_{23} / E_{0}=0.639 \\
\rho_{i} / \rho_{s} & =0.001362
\end{aligned}
$$

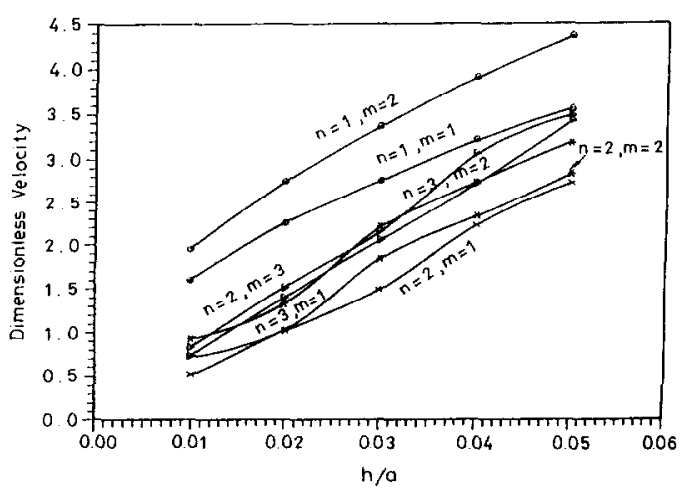

Fig. 7. Effect of $h / a$ on the dimensionless critical velocities of a $\left[(0)_{4}\right]$ laminated cylindrical shell when the natural frequencies vanish.

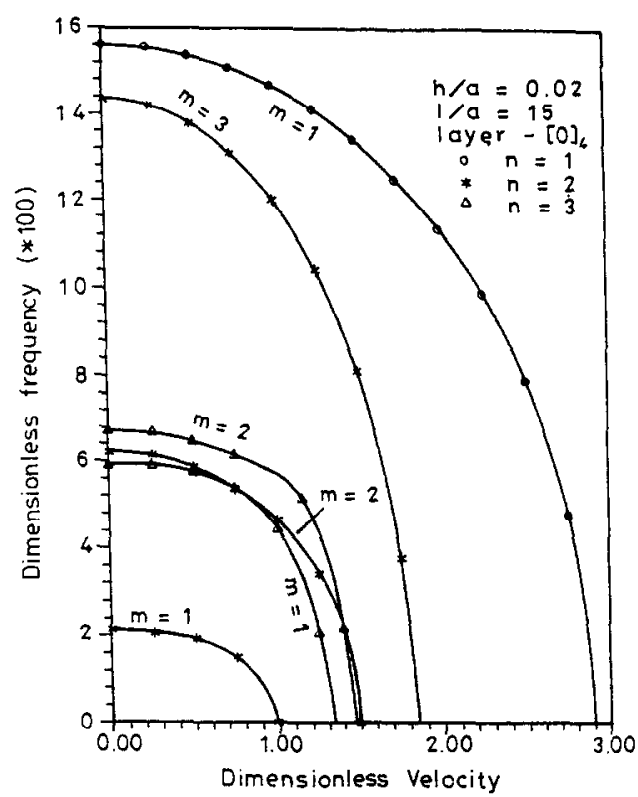

Fig. 8. Relationship of the dimensionless natural frequency vs dimensionless velocity of $\left[(0)_{4}\right]$ cylindrical shell with $h / a=0.02$ and $l / a=15$.

In addition, the clamped boundary conditions at both ends are used

$$
\begin{aligned}
& \bar{u}^{0}=0 \\
& \bar{v}^{0}=0 \\
& \bar{u}^{a}=0
\end{aligned}
$$

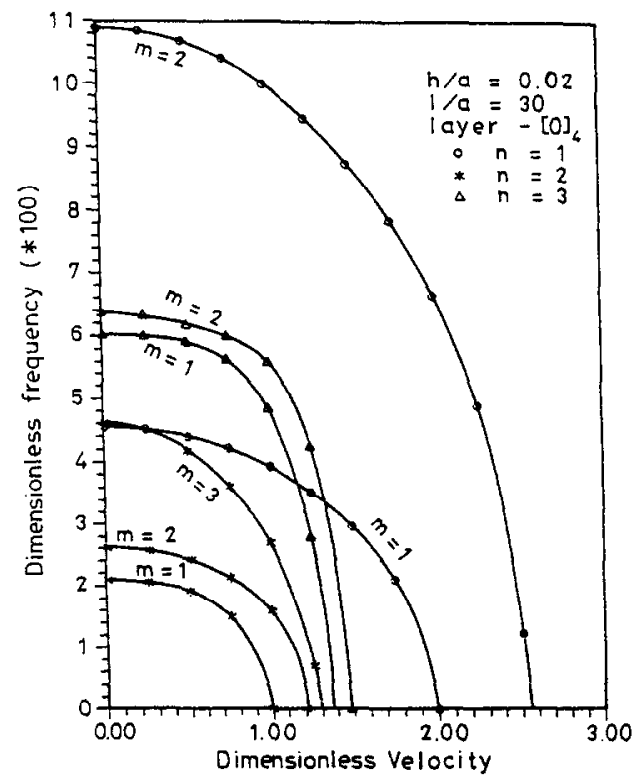

Fig. 9. Relationship of the dimensionless natural frequency vs dimensionless velocity of a $\left[(0)_{4}\right]$ cylindrical shell with $h / a=0.02$ and $/ / a=15$. 


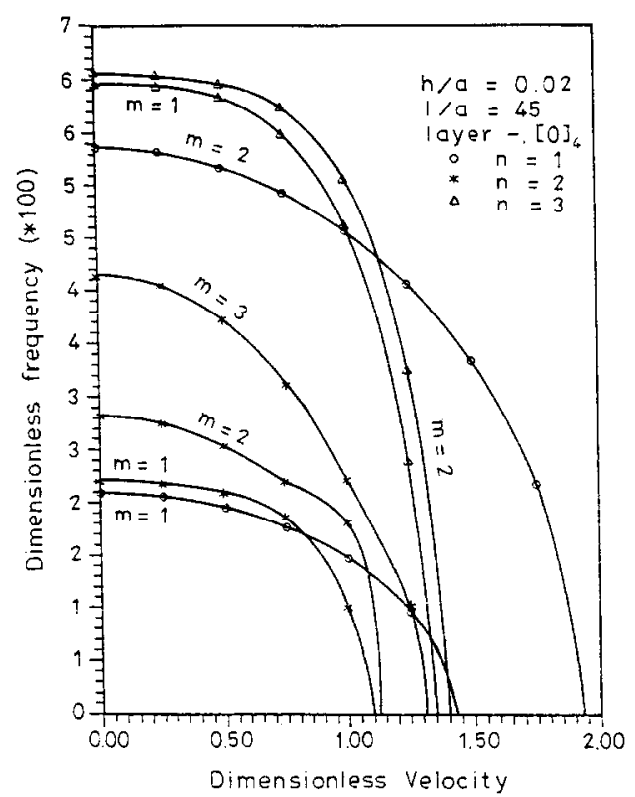

Fig. 10. Relationship of the dimensionless natural frequency vs dimensionless velocity of a $\left[(0)_{4}\right]$ cylindrical shell with $h / a=0.02$ and $l / a=15$.

$$
\begin{aligned}
\bar{v}^{a} & =0 \\
\bar{w}^{0} & =0 .
\end{aligned}
$$

The results are presented in Figs 1-18, which are discussed below. In the figures $m=1,2,3$ represents the first, second and third lowest fundamental frequencies.

\section{Effect of thickness-to-radius ratio}

Figures 1-5 show the relationship of the dimensionless natural frequencies vs dimensionless velocity of a $\left[(0)_{4}\right]$ cylindrical shell under various values of the thickness-to-ratio $h / a$. As it is already well known, the natural frequencies decrease as the fluid velocity increases. The circumferential mode $n=2$ (which corresponds to an elliptical shape of the deformed

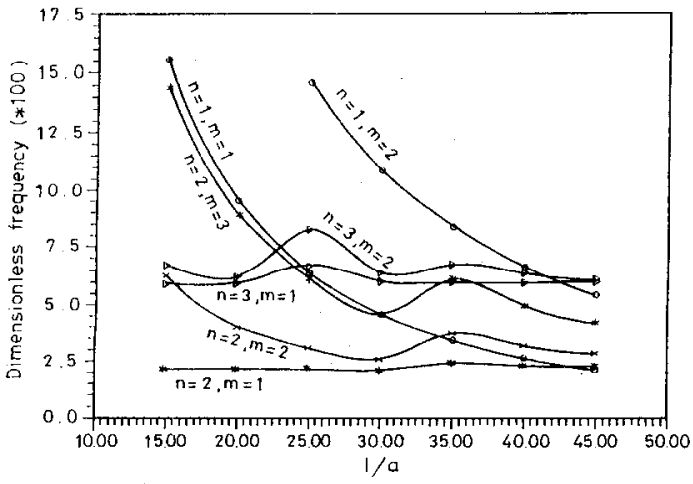

Fig. 11. Effects of $l / a$ on the dimensionless natural frequencies at zero fluid velocity.

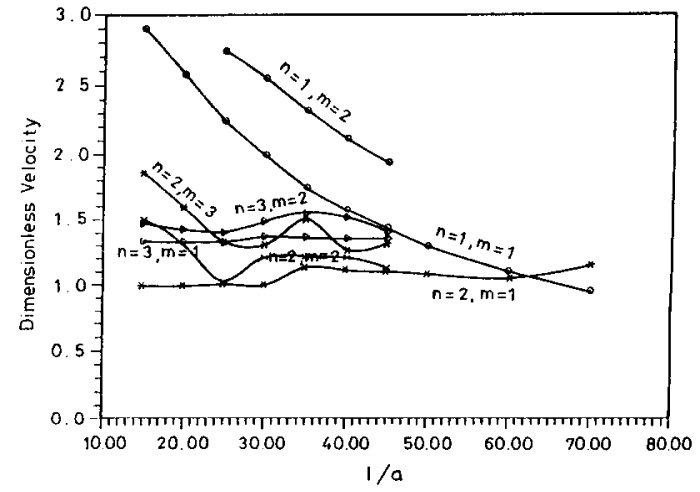

Fig. 12. Effect of $l / a$ on the dimensionless natura frequencies on the dimensionless critical velocity when the natural frequencies vanish.

cross-section) is the dominant mode. Figure 6 shows the effect of $h / a$ on the dimensionless frequencies when the fluid velocity is zero; in contrast, Fig. 7 shows the effect of $h / a$ on the dimensionless critical velocities when the natural frequencies vanish. It is seen from Fig. 6 that the natural frequencies are affected more by the shell thickness when there are more circumferential deformation (larger $n$ ). In the case of $n=1$, which corresponds to rigid body movement of the cross-section, the thickness has almost no effect on the dimensionless natural frequencies. In contrast, all of the dimensionless critical velocities are affected by the ratio $h / a$ as revealed in Fig. 7 .

\section{Effect of length-to-radius ratio}

Figures 8-10 show the relationship of the dimensionless natural frequencies vs dimensionless

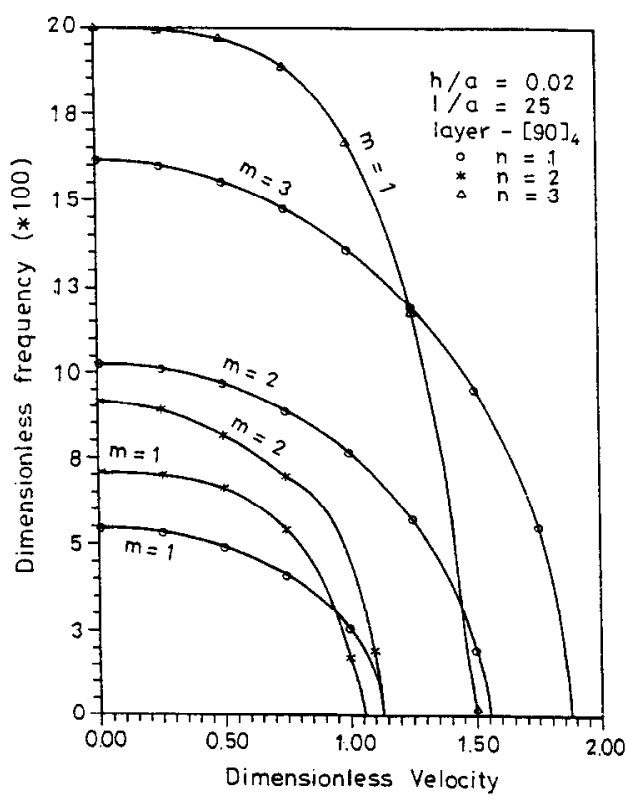

Fig. 13. Relationship of the dimensionless natural frequency vs dimensionless velocity of a $\left[(90)_{4}\right]$ cylindrical shell with $h / a=0.02$ and $l / a=25$. 


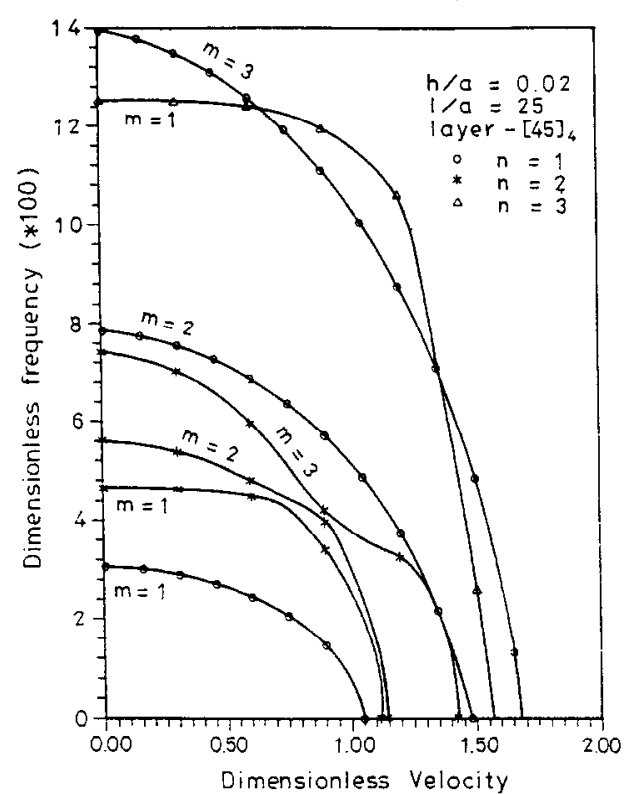

Fig. 14. Relationship of the dimensionless natural frequency vs dimensionless velocity of a $\left[(45)_{4}\right]$ cylindrical shell with $h / a=0.02$ and $l / a=25$.

velocity of $\left[(0)_{4}\right]$ cylindrical shell with $h / a=0.02$ under various values of the length-to-radius ratio $l / a$. As the figures show, the dimensionless natural frequency curves corresponding to various modes get closer as the value of $l / a$ becomes larger. In the case of $l / a=45$, the lowest natural frequency switches its mode from $n=2$ to $n=1$ near the value 0.65 of the dimensionless fluid velocity. Similar to Figs 6 and 7, Figs 11 and 12 summarize the effects of $l / a$ on the dimensionless natural frequency at zero fluid velocity

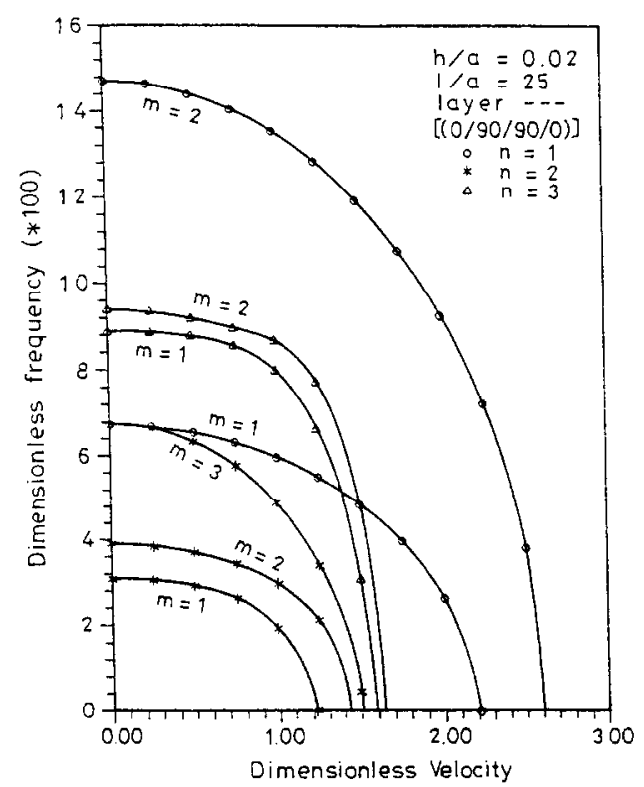

Fig. 15. Relationship of the dimensionless natural frequency vs dimensionless velocity of $[(0 / 90 / 90 / 0)]$ cylindrical shell with $h / a=0.02$ and $l / a=25$.

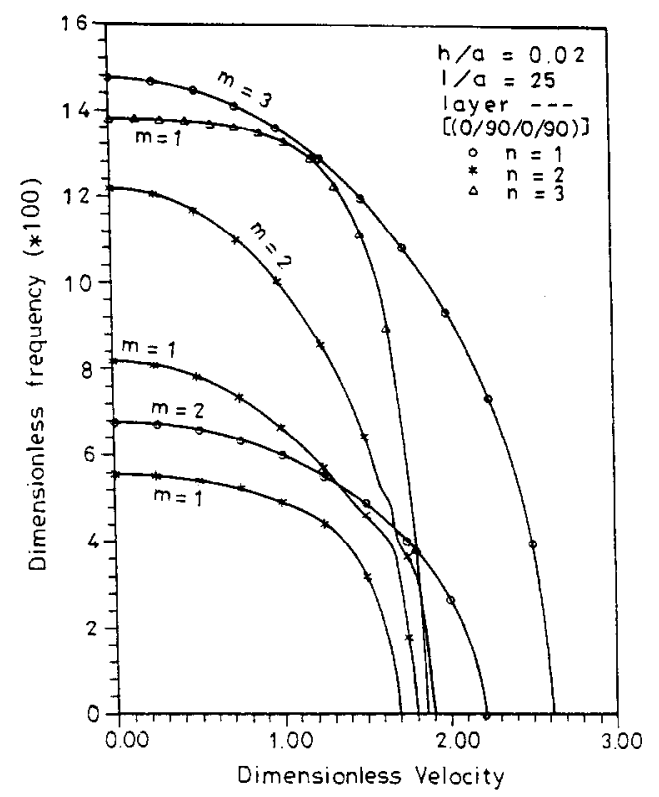

Fig. 16. Relationship of the dimensionless natural frequency vs dimensionless velocity of a $[(0 / 90 / 90 / 0)]$ cylindrical shell with $h / a=0.02$ and $l / a=25$.

and on the dimensionless critical velocity. In contrast to the monotone effects of $h / a$, it is shown that the effects of $l / a$ are more complex and depend on each individual mode.

\section{Effect of stacking angle}

Figures 13 and 14 show the relationship of the dimensionless natural frequencies vs dimensionless velocity of $\left[(90)_{4}\right]$ and $\left[(45)_{4}\right]$ cylindrical shell with $h / a=0.02$ and $l / a=25$. Compared to Fig. 2, these

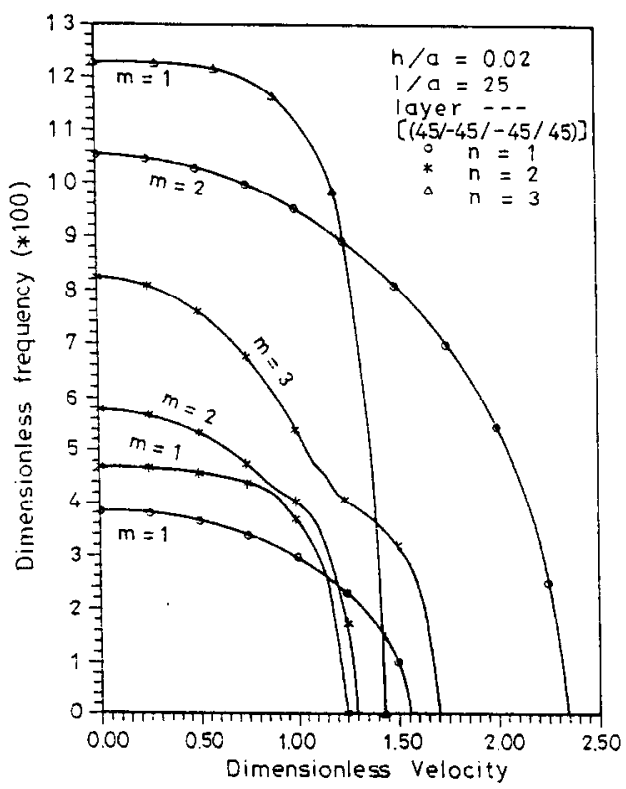

Fig. 17. Relationship of the dimensionless natural frequency vs dimensionless velocity of a $[(45 /-45 /-45 / 45)]$ cylindrical shell with $h / a=0.02$ and $l / a=25$. 


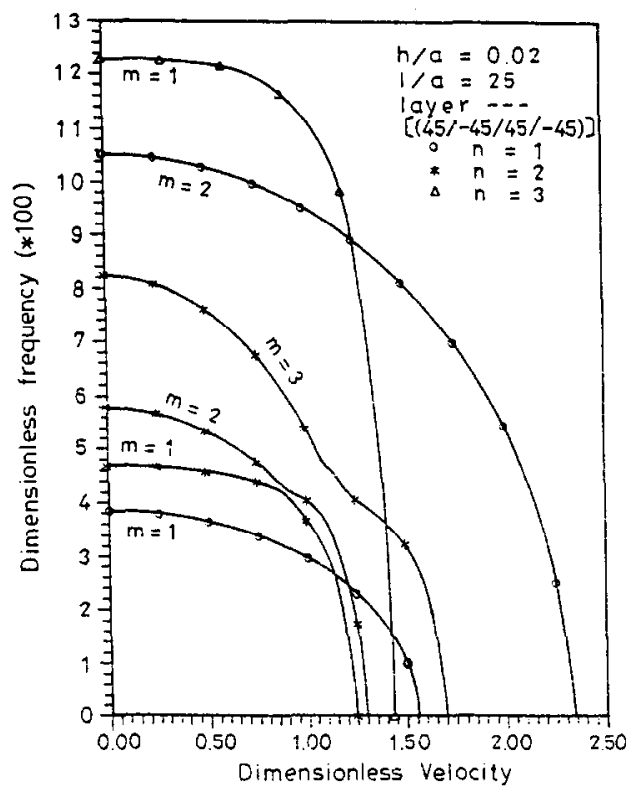

Fig. 18. Relationship of the dimensionless natural frequency vs dimensionless velocity of a $[(45 /-45 /-45 / 45)]$ cylindrical shell with $h / a=0.02$ and $l / a=25$.

two figures show the effects of strengthening in the circumferential direction and weakening in the longitudinal direction due to the layup angle. This is particularly noticeable when $n=1$ becomes the dominant mode of $\left[(45)_{4}\right]$ layup and partially dominant mode of $\left[(90)_{4}\right]$ layup. The results of the other more complex stackings are presented in Figs 15-18.

\section{CONCLUDING REMARKS}

The natural frequencies and critical velocities of laminated circular cylindrical shells with fixed-fixed ends conveying fluids are studied based on a transverse shear deformable theory for the moderately thick cylindrical shells. Numerical examples are presented, in which the parameter studies include stacking angle, length-to-radius and thickness-to-radius ratios. It is found that the thickness-to-radius ratio has a monotone effect on the natural frequencies and critical velocities; in contrast, the length-to-radius ratio has more complex effects on the natural frequencies and critical velocities. Finally, as expected, the layup angle changes the stiffness of the shell and hence the natural frequencies as well as the critical velocities and further, the mode of the lowest fundamental frequencies.

Acknowledgement - The authors wish to thank the National Science Council, Republic of China, for their financial support under the contract no. NSC-80-0401-E-002-07.

\section{REFERENCES}

1. S. S. Chen. Flow-induced Vibration of Circular Cylindrical Structures. Hemisphere, Washington, DC (1987).
2. R. W. Gregory and M. P. Païdossis, Unstable oscillation of tubular cantilevers conveying fluid, part I. Theory. Proc. R. Soc. A293, 512-527.

3. R. W. Gregory and M. P. Paidossis, Unstable oscillation of tubular cantilevers conveying fluid, part II. Experiment. Proc. R. Soc. A293, 528-542 (1966).

4. M. P. Païdossis, Dynamics of tubular cantilevers conveying fluid. J. Mech. Engng Sci. 12, 85-103 (1970).

5. M. P. Paîdossis, Flutter of conservative system of pipes conveying incompressible fluid. J. Mech. Engng Sci. 17, 19-25 (1975). Hydroelastic instability of uniformly curved pipe-fluid systems, 37, 817-822.

6. M. P. Païdossis and N. T. Issid, Dynamic stability of pipes conveying fluid. J. Sound Vibr. 33, 267-294 (1974).

7. S. S. Chen, Out-of-plane vibration and stability of curved tubes conveying fluid. J. appl. Mech. 40, 362-368 (1973).

8. B. E. Laithier and M. P. Paidossis, The equations of motion of initially stressed Timoshenko tubular beams conveying fluid. J. Sound Vibr. 79, 175-195 (1981).

9. T. L. C. Chen and C. W. Bert, Dynamic stability of isotropic or composite-material cylindrical shells containing swirling fluid flow. J. appl. Mech. 99, 112-116 (1977).

10. J. M. Clinch, Prediction and measurement of the vibration induced in the thin-walled pipes by the passage of internal turbulent water flow. $J$. Sound Vibr. 12, 429-451 (1970).

11. D. S. Weaver and B. Mylatun, On the stability of thin pipes with an internal flow. J. Sound. Vibr. 31, 399-410 (1953).

12. D. S. Weaver and B. Mylatun, On the stability of thin pipes with an internal flow. J. Sound Vibr. 31, 399-410 (1953).

13. M. P. Païdossis and J. P. Denise, Flutter of thin cylindrical shells conveying fluid. J. Sound Vibr. 20, 9-26 (1972).

14. S. S. Chen and G. S. Rosenberg, Free vibration and stability of fluid-conveying cylindrical shells. Trans. ASME J. Engng Indus. 96, 420-426 (1974).

15. D. S. Weaver and M. P. Paidossis, On the collapse and flutter phenomena in the tubes conveying fluid. J. Sound Vibr. 50, 117-132 (1977).

16. P. J. Holmes, Pipes supported at both ends cannot flutter. J. appl. Mech. 45, 619-622 (1978).

17. J. R. Vinson and R. L. Sierakowski, The Behavior of Structures Composed of Composite Materials. Martinus Nijhoff, Amsterdam (1986).

18. E. B. Magrab, Vibrations of Elastic Structural Members. Sijthoff \& Noordhoff, Alphen aan den Rijn (1979).

APPENDIX

$$
\begin{aligned}
a_{11}= & -\frac{\lambda^{2} Q_{11}}{a}-\frac{\lambda n Q_{16}}{a}-\frac{\lambda^{2} Q_{11} z}{a^{2}}-\frac{\lambda n Q_{16}}{a\left(1+\frac{z}{a}\right)}-\frac{Q_{66} n^{2}}{a\left(1+\frac{z}{a}\right)} \\
& -\frac{\lambda n Q_{16} z}{a^{2}\left(1+\frac{z}{a}\right)}+a \Omega^{2}+z \Omega^{2}
\end{aligned}
$$

$a_{12}=-\frac{\lambda^{2} Q_{11} z}{a}-\frac{\lambda n Q_{16} z}{a}-\frac{\lambda^{2} Q_{11} z^{2}}{a^{2}}-\frac{\lambda n Q_{16} z}{a\left(1+\frac{z}{a}\right)}$

$$
-\frac{Q_{66} n^{2} z}{a\left(1+\frac{z}{a}\right)}-\frac{\lambda n Q_{16} z^{2}}{a^{2}\left(1+\frac{z}{a}\right)}+a \Omega^{2} z+z^{2} \Omega^{2}
$$


938

Jeng-Shian Chang and Wen-Jiann Chiou

$$
\begin{aligned}
a_{13}= & -\frac{\lambda^{2} Q_{16} z}{a}-\frac{\lambda n Q_{66} z}{a}-\frac{\lambda^{2} Q_{16} z^{2}}{a^{2}}-\frac{\lambda n Q_{12} z}{a\left(1+\frac{z}{a}\right)} \\
& -\frac{Q_{26} n^{2} z}{a\left(1+\frac{z}{a}\right)}-\frac{\lambda n Q_{12} z^{2}}{a^{2}\left(1+\frac{z}{a}\right)} \\
a_{14}= & -\frac{\lambda^{2} Q_{16} z}{a}-\frac{\lambda n Q_{66} z}{a}-\frac{\lambda^{2} Q_{16} z^{2}}{a^{2}}-\frac{\lambda n Q_{12} z}{a\left(1+\frac{z}{a}\right)} \\
& -\frac{Q_{26} n^{2} z}{a\left(1+\frac{z}{a}\right)}-\frac{\lambda n Q_{12} z^{2}}{a^{2}\left(1+\frac{z}{a}\right)}
\end{aligned}
$$$$
a_{15}--\frac{i n Q_{26}}{a\left(1+\frac{z}{a}\right)}-\frac{i \lambda Q_{12}}{a\left(1+\frac{z}{a}\right)}-\frac{\lambda Q_{12} z}{a^{2}\left(1+\frac{z}{a}\right)}
$$$$
\begin{aligned}
a_{21}= & -\frac{\lambda^{2} Q_{11} z}{a}-\frac{\lambda n Q_{16} z}{a}-\frac{\lambda^{2} Q_{11} z^{2}}{a^{2}}-\frac{\lambda n Q_{16} z}{a\left(1+\frac{z}{a}\right)} \\
& -\frac{Q_{66} n^{2} z}{a\left(1+\frac{z}{a}\right)}-\frac{\lambda n Q_{16} z^{2}}{a^{2}\left(1+\frac{z}{a}\right)}+a \Omega^{2} z+z^{2} \Omega^{2}
\end{aligned}
$$$$
a_{22}=-\frac{\lambda^{2} Q_{11} z^{2}}{a}-\frac{\lambda n Q_{16} z^{2}}{a}-\frac{\lambda^{2} Q_{11} z^{3}}{a^{2}}-\frac{\lambda n Q_{16} z^{2}}{a\left(1+\begin{array}{l}
z \\
a
\end{array}\right)}
$$$$
-\frac{Q_{66} n^{2} z^{2}}{a\left(1+\frac{z}{a}\right)}-\frac{\lambda n Q_{16} z^{3}}{a^{2}\left(1+\frac{z}{a}\right)}+\Omega^{2} z^{3}+a z^{2} \Omega^{2}
$$$$
a Q_{5} 5-Q_{5} 5 z
$$

$$
\begin{aligned}
a_{23}= & -\frac{\lambda^{2} Q_{16} z}{a}-\frac{\lambda n Q_{66} z}{a}-\frac{\lambda^{2} Q_{16} z^{2}}{a^{2}}-\frac{\lambda n Q_{12} z}{a\left(1+\frac{z}{a}\right)} \\
& -\frac{Q_{26} n^{2} z}{a\left(1+\frac{z}{a}\right)}-\frac{\lambda n Q_{12} z^{2}}{a^{2}\left(1+\frac{z}{a}\right)}-\frac{Q_{45} z}{a\left(1+\frac{z}{a}\right)}-\frac{Q_{45} a}{a\left(1+\frac{z}{a}\right)} \\
a_{24}= & -\frac{\lambda^{2} Q_{16} z^{2}}{a}-\frac{\lambda n Q_{66} z^{2}}{a}-\frac{\lambda^{2} Q_{16} z^{3}}{a^{2}}-\frac{\lambda n Q_{12} z^{2}}{a\left(1+\frac{z}{a}\right)} \\
& -\frac{Q_{26} n^{2} z^{2}}{a\left(1+\frac{z}{a}\right)}-\frac{\lambda n Q_{12} z^{3}}{a^{2}\left(1+\frac{z}{a}\right)}-\frac{Q_{45} z}{\left(1+\frac{z}{a}\right)}-\frac{Q_{45} a}{\left(1+\frac{z}{a}\right)} \\
a_{25}= & -\frac{i \lambda Q_{55} z}{a}-i \lambda Q_{55}-\frac{i n Q_{45} a}{a\left(1+\frac{z}{a}\right)}+\frac{i \lambda Q_{12} z}{a\left(1+\frac{z}{a}\right)} \\
& +\frac{i n Q_{26} z}{a\left(1+\frac{z}{a}\right)}-\frac{i Q_{45} n z}{a\left(1+\frac{z}{a}\right)}+\frac{i \lambda Q_{12} z^{2}}{a^{2}\left(1+\frac{z}{a}\right)}
\end{aligned}
$$

$$
\begin{gathered}
a_{31}=-\frac{\lambda^{2} Q_{16} z}{a}-\frac{\lambda n Q_{12}}{a}-\frac{\lambda^{2} Q_{16} z}{a}-\frac{\lambda n Q_{66}}{a\left(1+\frac{z}{a}\right)} \\
-\frac{Q_{26} n^{2}}{a\left(1+\frac{z}{a}\right)}-\frac{\lambda n Q_{66} z}{a^{2}\left(1+\frac{z}{a}\right)}
\end{gathered}
$$

$$
\begin{aligned}
a_{32}= & -\frac{\lambda^{2} Q_{16} z}{a}-\frac{\lambda n Q_{12} z}{a}-\frac{\lambda^{2} Q_{16} z}{a}-\frac{\lambda n Q_{66} z}{a\left(1+\frac{z}{a}\right)} \\
& -\frac{Q_{26} n^{2} z}{a\left(1+\frac{z}{a}\right)}-\frac{\lambda n Q_{66} z^{2}}{a^{2}\left(1+\frac{z}{a}\right)}-Q_{45}
\end{aligned}
$$

$$
\begin{aligned}
a_{33}= & -\frac{\lambda^{2} Q_{66}}{a}-\frac{\lambda n Q_{26}}{a}-\frac{\lambda^{2} Q_{66} z}{a^{2}}-\frac{\lambda n Q_{26}}{a\left(1+\frac{z}{a}\right)}-\frac{Q_{22} n^{2}}{a\left(1+\frac{z}{a}\right)} \\
& -\frac{\lambda n Q_{26} z}{a^{2}\left(1+\frac{z}{a}\right)}-\frac{Q_{45}}{a\left(1+\frac{z}{a}\right)}+a \Omega^{2}+\Omega^{2} z
\end{aligned}
$$$$
\begin{aligned}
a_{34}= & -\frac{\lambda^{2} Q_{66} z}{a}-\frac{\lambda n Q_{26} z}{a}-\frac{\lambda^{2} Q_{66} z^{2}}{a^{2}}-\frac{i n Q_{26} z}{a\left(1+\frac{z}{a}\right)} \\
& -\frac{Q_{22} n^{2} z}{a\left(1+\frac{z}{a}\right)}-\frac{\lambda n Q_{26} z^{2}}{a^{2}\left(1+\frac{z}{a}\right)}-\frac{Q_{44}}{\left(1+\frac{z}{a}\right)}+a \Omega^{2} z+\Omega^{2} z^{2}
\end{aligned}
$$

$$
\begin{aligned}
a_{35}= & \frac{i \lambda Q_{45} z}{a}+\frac{i n Q_{22}}{a\left(1+\frac{z}{a}\right)}+\frac{i \lambda Q_{26}}{a\left(1+\frac{z}{a}\right)}+\frac{i n Q_{44}}{a\left(1+\frac{z}{a}\right)} \\
& +\frac{i Q_{26} \lambda=}{a\left(1+\frac{z}{a}\right)}
\end{aligned}
$$

$$
\begin{aligned}
a_{41}= & -\frac{\lambda^{2} Q_{16} z}{a}-\frac{\lambda n Q_{12} z}{a}-\frac{\lambda^{2} Q_{16} z^{2}}{a^{2}}-\frac{\lambda n Q_{66} z}{a\left(1+\frac{z}{a}\right)} \\
& -\frac{Q_{26} n^{2} z}{a\left(1+\frac{z}{a}\right)}-\frac{\lambda n Q_{66} z_{2}}{a^{2}\left(1+\frac{z}{a}\right)}
\end{aligned}
$$

$$
\begin{aligned}
a_{42}= & -\frac{\lambda^{2} Q_{16} z^{2}}{a}-\frac{\lambda n Q_{12} z^{2}}{a}-\frac{\lambda^{2} Q_{16} z^{3}}{a^{2}}-\frac{\lambda n Q_{66} z^{2}}{a\left(1+\frac{z}{a}\right)} \\
& -\frac{Q_{26} n^{2} z^{2}}{a\left(1+\frac{z}{a}\right)}-\frac{\lambda n Q_{66} z^{3}}{a^{2}\left(1+\frac{z}{a}\right)}-Q_{45} a
\end{aligned}
$$

$a_{43}=-\frac{\lambda^{2} Q_{66} z}{a}-\frac{\lambda n Q_{26} z}{a}-\frac{\lambda^{2} Q_{66} z^{2}}{a^{2}}-\frac{\lambda n Q_{26} z}{a\left(1+\frac{z}{a}\right)}-\frac{\lambda n Q_{22} z}{a\left(1+\frac{z}{a}\right)}$

$$
+\frac{\lambda n Q_{26} z^{2}}{a^{2}\left(1+\frac{z}{a}\right)}-\frac{Q_{44}}{\left(1+\frac{z}{a}\right)}+a \Omega^{2} z+\Omega^{2} z^{2}
$$


Fixed-fixed laminated cylindrical shells conveying fluids

939

$$
\begin{aligned}
a_{44}= & -\frac{\lambda^{2} Q_{66} z}{a}-\frac{\lambda n Q_{26} z^{2}}{a}-\frac{\lambda^{2} Q_{66} z^{2}}{a}-\frac{\lambda n Q_{26} z^{2}}{a\left(1+\frac{z}{a}\right)} \\
& -\frac{Q_{22} n^{2} z^{2}}{a\left(1+\frac{z}{a}\right)}-\frac{\lambda n Q_{26} z^{3}}{a^{2}\left(1+\frac{z}{a}\right)}-\frac{Q_{44} a}{\left(1+\frac{z}{a}\right)} \\
& +a \Omega^{2} z^{2}+\Omega^{2} z^{3} \\
a_{45}= & -i \lambda Q_{45}-\frac{i n Q_{22} z}{a\left(1+\frac{z}{a}\right)}+\frac{i \lambda Q_{26} z}{a\left(1+\frac{z}{a}\right)}+\frac{i n Q_{44} a}{a\left(1+\frac{z}{a}\right)} \\
& +\frac{i Q_{26} \lambda z^{2}}{a\left(1+\frac{z}{a}\right)} \\
a_{51}= & -\frac{i n Q_{26}}{a\left(1+\frac{z}{a}\right)}-\frac{i \lambda Q_{12}}{a} \\
a_{52}= & \frac{i \lambda Q_{55} z}{a}+i \lambda Q_{55}+i n Q_{45}-\frac{i \lambda Q_{12} z}{a}-\frac{i n Q_{26} z}{a\left(1+\frac{z}{a}\right)}
\end{aligned}
$$

$$
\begin{aligned}
a_{53}= & \frac{i \lambda Q_{45} z}{a}+\frac{i n Q_{22}}{a\left(1+\frac{z}{a}\right)}+\frac{i \lambda Q_{26}}{a\left(1+\frac{z}{a}\right)}+\frac{i n Q_{44}}{a\left(1+\frac{z}{a}\right)} \\
& +\frac{i Q_{26} \lambda z}{a\left(1+\frac{z}{a}\right)} \\
a_{54}= & -\frac{i n Q_{22} z}{a\left(1+\frac{z}{a}\right)}-\frac{i \lambda Q_{26} z}{a}+\frac{i n Q_{44}}{\left(1+\frac{z}{a}\right)}+\frac{i Q_{45} \lambda z}{a\left(1+\frac{z}{a}\right)} \\
& +\frac{i Q_{45} \lambda}{\left(1+\frac{z}{a}\right)} \\
a_{55}= & -\frac{\lambda n Q_{45}}{a}-\frac{\lambda^{2} Q_{55}}{a}-\frac{\lambda^{2} Q_{55}}{a^{2}}-\frac{Q_{22}}{a\left(1+\frac{z}{a}\right)}-\frac{\lambda n Q_{45} z}{a^{2}\left(1+\frac{z}{a}\right)} \\
& +a \Omega^{2}+z \Omega^{2}+\frac{a}{h} \frac{\rho_{i}}{\rho_{s}} \frac{(\Omega+O)^{2}}{n+\lambda I_{n+1}(\lambda) / I_{n}(\lambda)} .
\end{aligned}
$$

